\title{
Nerve transfer versus muscle transfer to restore elbow flexion after pan-brachial plexus injury: a cost-effectiveness analysis
}

\author{
Arvin R. Wali, BA, David R. Santiago-Dieppa, MD, Justin M. Brown, MD, and Ross Mandeville, MD \\ Department of Neurological Surgery, University of California, San Diego, California
}

OBJECTIVE Pan-brachial plexus injury (PBPI), involving C5-T1, disproportionately affects young males, causing lifelong disability and decreased quality of life. The restoration of elbow flexion remains a surgical priority for these patients. Within the first 6 months of injury, transfer of spinal accessory nerve (SAN) fascicles via a sural nerve graft or intercostal nerve (ICN) fascicles to the musculocutaneous nerve can restore elbow flexion. Beyond 1 year, free-functioning muscle transplantation (FFMT) of the gracilis muscle can be used to restore elbow flexion. The authors present the first costeffectiveness model to directly compare the different treatment strategies available to a patient with PBPI. This model assesses the quality of life impact, surgical costs, and possible income recovered through restoration of elbow flexion.

METHODS A Markov model was constructed to simulate a 25-year-old man with PBPI without signs of recovery 4.5 months after injury. The management options available to the patient were SAN transfer, ICN transfer, delayed FFMT, or no treatment. Probabilities of surgical success rates, quality of life measurements, and disability were derived from the published literature. Cost-effectiveness was defined using incremental cost-effectiveness ratios (ICERs) defined by the ratio between costs of a treatment strategy and quality-adjusted life years (QALYs) gained. A strategy was considered cost-effective if it yielded an ICER less than a willingness-to-pay of $\$ 50,000 / Q A L Y$ gained. Probabilistic sensitivity analysis (PSA) was performed to address parameter uncertainty.

RESULTS The base case model demonstrated a lifetime QALYS of 22.45 in the SAN group, 22.0 in the ICN group, 22.3 in the FFMT group, and 21.3 in the no-treatment group. The lifetime costs of income lost through disability and interventional/rehabilitation costs were $\$ 683,400$ in the SAN group, $\$ 727,400$ in the ICN group, $\$ 704,900$ in the FFMT group, and $\$ 783,700$ in the no-treatment group. Each of the interventional modalities was able to dramatically improve quality of life and decrease lifelong costs. A Monte Carlo PSA demonstrated that at a willingness-to-pay of $\$ 50,000 / Q A L Y$ gained, SAN transfer dominated in $88.5 \%$ of iterations, FFMT dominated in $7.5 \%$ of iterations, ICN dominated in $3.5 \%$ of iterations, and no treatment dominated in $0.5 \%$ of iterations.

CONCLUSIONS This model demonstrates that nerve transfer surgery and muscle transplantation are cost-effective strategies in the management of PBPI. These reconstructive neurosurgical modalities can improve quality of life and lifelong earnings through decreasing disability.

https://thejns.org/doi/abs/10.3171/2017.4.FOCUS17112

KEY WORDS cost-effectiveness; peripheral nerve surgery; pan-brachial plexus injury; nerve transfer surgery; spinal accessory nerve; intercostal nerve; gracilis muscle

$\mathrm{T}$ RAUMA to nerve roots C5-T1 caused by contusions, lacerations, gunshot wounds, penetrative injury, or traction injuries can cause pan-brachial plexus injury (PBPI) ${ }^{17,21}$ A significant subset of these patients have preganglionic avulsions that would preclude spontaneous recovery..$^{10,21}$ Adult patients with brachial plexus injury are disproportionately young males, with mean ages ranging from 25 to 29 years old, who can experience major disability and a lower quality of life after injury. ${ }^{20}$ While published surveys indicate that restoration of elbow flexion

ABBREVIATIONS CPT = Current Procedural Terminology; EMG = electromyography; FFMT = free-functioning muscle transplantation; ICER = incremental cost-effectiveness ratio; ICN = intercostal nerve; ICU = intensive care unit; $M R C=$ Medical Research Council; PBPI = pan-brachial plexus injury; PSA = probabilistic sensitivity analysis; $\mathrm{QALY}=$ quality-adjusted life year; SAN = spinal accessory nerve.

SUBMITTED March 1, 2017. ACCEPTED April 18, 2017.

INCLUDE WHEN CITING DOI: 10.3171/2017.4.FOCUS17112. 
remains a top surgical priority for patients and providers, the recommended interventions to achieve this goal vary broadly. 3,25

Restoration of elbow flexion has been demonstrated to improve quality of life and improve the likelihood an individual is able to rejoin the workforce and earn income.,12 The most common extraplexal nerve transfers used to restore elbow flexion in PBPI are transfer of the spinal accessory nerve (SAN) fascicles with sural nerve grafts or intercostal nerve (ICN) fascicles without nerve grafts to the musculocutaneous nerve..$^{30}$ Nerve transfer surgery is ideally performed within 6 months of injury for optimal results. ${ }^{26}$ Reasons for delay in presentation for reconstructive neurosurgery may include delayed diagnosis, lack of awareness about reconstructive techniques, failed prior surgery, or prolonged waiting for spontaneous recovery. ${ }^{13}$ Patients who undergo surgical treatment to restore elbow flexion after this window of time may undergo muscle transplantation rather than nerve transfer to restore function. ${ }^{14}$ Free-functioning muscle transplantation (FFMT) involves transplantation of a muscle, commonly the gracilis muscle, to the clavicle and the distal biceps tendon to restore elbow flexion..$^{2,13,24}$ The gracilis muscle transplant is most commonly innervated by the SAN or ICNs. ${ }^{2}$ While efficacious in achieving adequate elbow function, FFMT exposes patients to additional risks such as possible thrombosis of the newly anastomosed vessels for the gracilis muscle, which may result in graft failure, reoperations, and prolonged hospitalizations with extensive intensive care unit (ICU) monitoring. Both nerve transfer surgery and FFMT are reconstructive neurosurgical options available to restore elbow flexion.

While prior studies have reported surgical success rates to restore elbow flexion in PBPI using SAN transfer, ICN transfer, or FFMT, this study presents the first costeffectiveness model to calculate the ratio of health utility recovered through different treatment options by the costs of intervention. This model captures the choices available to a 25-year-old-patient who sustained PBPI with preganglionic avulsions and is without any signs of neurological recovery 4.5 months after injury. The patient is considering treatment with nerve transfer surgery via the SAN transfer or the ICN transfer within 6 months of injury, delayed treatment with FFMT (more than 1 year after injury), or no treatment to restore elbow flexion. This model provides the first quantification of quality of life impact and resource utilization across the differing treatment options for PBPI. This model demonstrates the capacity of these underutilized and time-sensitive reconstructive neurosurgical techniques to restore functionality and optimize quality of life.

\section{Methods \\ Treatment Strategies}

Our cost-effectiveness model captures the decision to undergo early nerve transfer surgery, delayed FFMT, or no treatment after sustaining a PBPI. Patients who undergo nerve transfer surgery may select between SAN transfer with sural grafts or ICN transfer to the musculocutaneous nerve to restore elbow flexion. Patients who undergo single FFMT will have innervation of this transplanted muscle by either the SAN or ICN. The competing treatment strategies in our models were as follows (Fig. 1): 1) SAN transfer with sural nerve graft within 6 months after injury (Strategy 1); 2) ICN transfer within 6 months after injury without a nerve graft (Strategy 2); 3) gracilis muscle transplant innervated by either SAN or ICN 1 year after injury, without a nerve graft in both cases (Strategy 3); and 4) no surgical treatment to restore elbow flexion at any point in time (Strategy 4).

\section{Decision Model}

This decision-analysis model calculates the cost-effectiveness of the competing treatment strategies listed above. The role of additional surgeries to restore shoulder function or hand flexion was not included within this model. Data from the published literature were collected to provide model inputs for surgical success rates, complication rates, and quality of life information tabulated as quality-adjusted life years (QALYs) to model health utility across a lifetime. QALY values range from 0 to 1 , in which 1 refers to a full year of life in complete health. ${ }^{28}$ Complication rates such as surgical failure or thrombosis of transplanted vessels were modeled as causing long-term deficits in elbow flexion resulting in poorer quality of life. A Markov model was used to investigate the base scenario of a 25-year-old patient with 78 years of life expectancy ${ }^{6}$ with PBPI.

\section{Model Inputs \\ Neurological Strength Outcome}

Different grades of elbow flexion, defined by the British Medical Research Council (MRC), were chosen as the primary clinical outcome. ${ }^{7,23,29}$ MRC grades corresponding to neurological strength were grouped into good, fair, and poor. Patients with good outcome had MRC Grade 4-5, corresponding to elbow flexion against gravity with resistance. Patients with fair outcome had MRC Grades 2-3, corresponding to elbow flexion in the absence of gravity or in the presence of gravity without resistance. Patients with poor outcome had MRC Grade $0-1$, corresponding to the inability to flex the elbow without resistance in the absence of gravity. QALYs associated with good, fair, and poor outcomes with elbow flexion have been described in prior studies and correspond to values of $0.89,0.82$, and 0.81 QALYs, respectively. ${ }^{1}$

\section{Model Probabilities}

\section{MRC Grade by Type of Treatment}

The base model assumes that all individuals with PBPI have persistent unilateral MRC Grade 0 elbow flexion 4.5 months after injury. Consequently, our model assumed that patients with PBPI with preganglionic avulsions from C-5 to T-1 or with ruptures and no signs of spontaneous recovery were not subject to achieving improvements in elbow flexion without surgical intervention involving either nerve transfer surgery or FFMT. ${ }^{14}$

According to published data on nerve transfers for PBPI, both SAN transfer and the ICN transfer are efficacious in achieving elbow flexion. Merrell et al. conducted a 


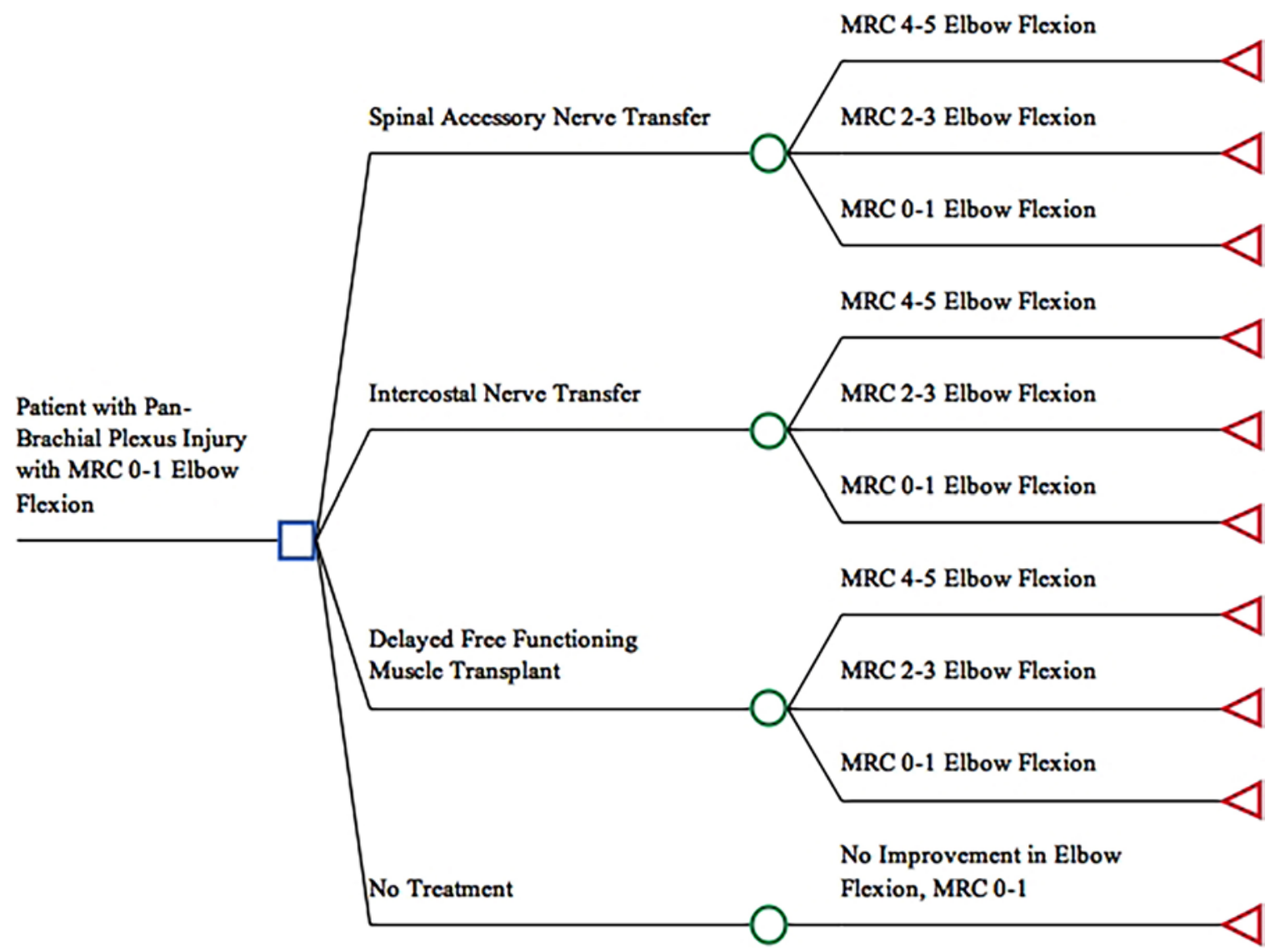

FIG. 1. The decision analysis model captures management options available to a patient with PBPI seeking to restore elbow flexion. Patients may undergo SAN transfer with sural grafts, ICN transfer, delayed FFMT, or no treatment. After surgery, patients either have good outcome defined by MRC Grade 4-5 elbow flexion, fair outcome defined by MRC Grade 2-3 elbow flexion, or poor outcome defined by MRC Grade $0-1$ elbow flexion.

robust meta-analysis investigating the MRC grade for 965 patients with brachial plexus avulsion, which ranged from C5-6 to PBPI, who underwent nerve transfer surgery. ${ }^{19}$ Thirty-nine percent of the 965 patients in the analysis by Merrell et al. underwent SAN transfer, of whom $29 \%$ of patients achieved MRC Grade 4-5. In contrast, 54\% of the 965 patients in the analysis by Merrell et al. underwent ICN transfer. Of the patients who underwent ICN transfer and did not require additional nerve grafting surgery, 41\% achieved MRC Grade 4-5. Comparably, Waikakul et al. conducted a prospective randomized parallel trial of 205 patients who either underwent SAN transfer or ICN transfer for restoration of elbow flexion within 6 months of injury with confirmed complete palsy of the brachial plexus. ${ }^{30}$ One hundred thirty patients underwent SAN nerve transfer with sural nerve grafts to restore elbow flexion. Forty-three percent of patients achieved MRC Grade $4-5,55 \%$ of patients achieved MRC Grade 2-3, and 2\% of patients experienced surgical failure and remained at MRC Grade 0-1 at the 2-year follow-up. Seventy-five patients underwent ICN transfer to restore elbow flexion, $20 \%$ of whom achieved MRC Grade 4-5, 73\% achieved MRC Grade 2-3, and 7\% experienced surgical failure and remained at MRC Grade 0-1 at the 2-year follow-up. Nagano et al. reported similar outcomes for ICN transfer to restore elbow flexion in a retrospective review of 35 pa- tients with PBPI: $26 \%$ of patients achieved MRC Grade $4-5,69 \%$ of patients achieved MRC Grade 2-3, and 5\% of patients experienced surgical failure with MRC Grade $0-1 .{ }^{22}$ Given the comparable rates of success for SAN and ICN transfer, our base model used the success rates from the prospective data reported by Waikakul et al., which had strict inclusion criteria for all patients having PBPI and intervention within 6 months of injury, to model SAN and ICN transfer success rates.

Published literature describing success rates after FFMT of a single gracilis muscle report similar outcomes in restoring elbow flexion. Maldonado et al. retrospectively reported 31 patients with PBPI who underwent a single gracilis muscle transplant innervated by either the SAN or the ICN..$^{18}$ Overall, FFMT resulted in $39 \%$ of patients with MRC Grade $4-5,55 \%$ of patients with MRC Grade $2-3$, and $6 \%$ of patients who remained at MRC Grade $0-1$. While patients who have a SAN donor to the gracilis muscle may have improved outcomes compared with ICN donors ${ }^{27}$ Maldonado et al. reports that $70 \%$ of patients had an MRC grade $\geq 3$ with SAN donors compared with $60 \%$ of patients with an MRC grade $\geq 3$ with ICN donors, in which there was no statistically significant difference in outcomes between these groups. Yang et al. reported a case series of 47 patients in which 2 patients required additional gracilis surgery in the case of vessel thrombosis, 
with final outcomes of $50 \%$ of patients achieving MRC Grade 4-5.32 Our base model used the success rates reported by Maldonado et al. because these patients had the gracilis muscle innervated by either the SAN or ICN and did not undergo additional surgery.

\section{Disability by Type of Treatment}

An analysis by Felici et al. demonstrated that $60 \%$ of patients with PBPI had disability and thus were receiving compensation from insurance companies. ${ }^{12} \mathrm{Of}$ the patients with PBPI, recovery of elbow function resulted in $15 \%$ of patients no longer being classified as disabled. To model these changes in disability status after surgery within our model, patients who achieved MRC Grade 4-5 for elbow flexion after surgery had a $15 \%$ increased probability of returning to the workforce. Using these values, patients who had MRC Grade $0-3$ had a $60 \%$ disability rate while patients with MRC Grade 4-5 had a $45 \%$ disability rate. This, in turn, corresponded to $40 \%$ of patients with MRC Grade $0-3$ returning to work while $55 \%$ of patients who achieved MRC Grade 4-5 returned to work. To model annual income lost through not returning to the workforce, mean annual wage in 2017 dollars (https://www.bls.gov/ data/inflation_calculator.htm) was calculated from the Bureau of Labor Statistics (https://www.bls.gov/oes/2015/ may/oes_nat.htm). Patients within the decision model had an annual chance of death from natural causes derived from US Census Bureau data (https://www.cdc.gov/nchs/ nvss/mortality_methods.htm).

\section{Costs by Type of Treatment}

All costs were modeled from the perspective of a thirdparty payer. Costs associated with surgery and health care services were based on the 2015 Medicare fee-for-service payment values utilizing Current Procedural Terminology (CPT) codes and adjusted to 2017 dollars (https://www. cms.gov/apps/physician-fee-schedule). Patients who underwent nerve transfer were subject to the direct costs of surgery, preoperative MRI and electromyography (EMG), anesthesia costs, neurosurgery consult costs, postoperative rehabilitation, and costs associated with inpatient hospital stay. Costs of inpatient hospital stay were calculated using published average costs per day for both inpatient and ICU stays (http://kff.org/health-costs/state-indicator/expensesper-inpatient-day). ${ }^{9}$ The Diagnosis-Related Group classification system was not used due to the variability in coding and lack of ability to reflect true cost differences incurred between the groups. Given the less invasive nature of SAN transfer compared with the other interventions, patients who had SAN nerve transfer had only 1 postoperative inpatient hospital day compared with 2 inpatient hospital days for the ICN group. Given shorter operations to conduct SAN nerve transfer, ${ }^{30}$ patients who had ICN transfer or FFMT had double the anesthesia costs. Patients who underwent SAN transfer had the additional cost of sural nerve grafting, and patients who underwent FFMT had the additional cost of flap transfer. Patients who underwent FFMT also had 2 days of postoperative ICU monitoring for vessel thrombosis in addition to 2 postoperative inpatient hospital days. Patients who chose not to undergo treatment did not incur any procedural or postoperative re- habilitation costs. Data on cost derivations associated with this analysis are described in Table 1. Data on probabilities of achieving each functional state, QALYs associated with each functional state, and total costs associated with each treatment strategy are presented in Table 2.

\section{Statistical Analysis}

TreeAge Pro 2016 (TreeAge Software) was used to perform all analyses for this model and to assess incremental cost-effectiveness ratios (ICERs). All health care utilities were discounted by an annual 3\% to adjust for future changes in quality of life..$^{16}$ Cost-effectiveness of the differing surgical treatment strategies and the no-treatment strategy were compared using ICERs, representing the ratio between the costs incurred with a given treatment strategy and QALYs gained by that strategy. A treatment strategy was considered cost-effective if the corresponding ICER was less than a standardized willingness-to-pay cutoff of $\$ 50,000 / \mathrm{QALY}$ gained. ${ }^{5} \mathrm{~A}$ treatment strategy was considered dominated by a more cost-effective option if it yielded either a negative ICER or a positive ICER greater than \$50,000/QALY gained. Base case analysis assessed the QALY gained after surgical intervention or no treatment while considering the costs associated with each treatment option and the income lost through persistent disability.

To adjust for parameter uncertainty, 1- and 2-way sensitivity analyses were performed. A Monte Carlo probabilistic sensitivity analysis (PSA) was performed using 100,000 iterations to capture distributions of all model inputs such as cost of surgery, surgical success rates, and rate of returning to work with good surgical outcome. ${ }^{8}$ Within the PSA, age parameters were given binary distributions, costs were modeled as gamma distributions ranging from zero to infinity, and probabilities and quality of life were modeled as beta distributions ranging from 0 to 1 . The only distributions not included within the PSA

\section{TABLE 1. Components of surgical costs}

\begin{tabular}{lrl}
\hline \multicolumn{1}{c}{ Direct Cost Component } & Fees $(\$)^{*}$ & CPT Code/Citation \\
\hline $\begin{array}{l}\text { Nerve transfer w/ sural nerve graft } \\
\text { facility fee + physician fee }\end{array}$ & 2858.72 & 64905,64893 \\
\hline Nerve transfer fee + physician fee & 2691.68 & 64905 \\
\hline Flap transfer fee + physician fee & 4797.64 & 15756,64905 \\
\hline Anesthesia & 686.46 & 1710 \\
\hline New neurosurgery clinic visit & 209.23 & 99205 \\
\hline Pre/postop surgical visits & 1000.30 & 99203 \\
\hline Nerve conduction study preop & 202.47 & 95910 \\
\hline EMG preop & 92.59 & 95886 \\
\hline MRI of cervical spine preop & 227.53 & 72141 \\
\hline Rehabilitation new visit & 87.67 & 97003 \\
\hline Rehabilitation follow-up & 380.04 & 97004 \\
\hline ICU daily cost & 4346.98 & Dasta et al., 2005 \\
\hline US inpatient hospital day & 2326.78 & Kaiser Family \\
& & Foundation \\
\hline
\end{tabular}

* Fees derived from 2015 Medicare fee-for-service payment values and adjusted to 2017 dollars. 
TABLE 2. Parameters used in cost-effectiveness model

\begin{tabular}{|c|c|c|c|c|}
\hline Model Parameters & Mean & SD & Distribution & Source (1st author or Medicare) \\
\hline Patient age (yrs) & 25 & 5 & Binomial & Waikakul et al., 1999 \\
\hline Annual wage (\$) & $49,506.92$ & 9901.38 & Gamma & BLS, 2015 data \\
\hline \multicolumn{5}{|l|}{ QALY MRC grade } \\
\hline $4-5$ & 0.89 & 0.11 & Beta & Ali et al., 2014 \\
\hline $2-3$ & 0.82 & 0.11 & Beta & Ali et al., 2014 \\
\hline $0-1$ & 0.81 & 0.14 & Beta & Ali et al., 2014 \\
\hline \multicolumn{5}{|l|}{ Model probabilities } \\
\hline \multicolumn{5}{|l|}{ MRC grade after SAN transfer } \\
\hline $4-5$ & 0.41 & 0.082 & Beta & Waikakul et al., 1999 \\
\hline $2-3$ & 0.55 & 0.11 & Beta & Waikakul et al., 1999 \\
\hline $0-1$ & 0.02 & 0.004 & Beta & Waikakul et al., 1999 \\
\hline \multicolumn{5}{|l|}{ MRC grade after ICN transfer } \\
\hline $4-5$ & 0.26 & 0.052 & Beta & Waikakul et al., 1999 \\
\hline $2-3$ & 0.69 & 0.138 & Beta & Waikakul et al., 1999 \\
\hline $0-1$ & 0.05 & 0.01 & Beta & Waikakul et al., 1999 \\
\hline \multicolumn{5}{|l|}{ MRC grade after FFMT } \\
\hline $4-5$ & 0.45 & 0.09 & Beta & Maldonado et al., 2016 \\
\hline $2-3$ & 0.45 & 0.09 & Beta & Maldonado et al., 2016 \\
\hline $0-1$ & 0.10 & 0.02 & Beta & Maldonado et al., 2016 \\
\hline Disability w/ MRC Grade 4-5 & 0.60 & 0.12 & Beta & Felici et al., 2014 \\
\hline Disability w/ MRC Grade 0-3 & 0.45 & 0.09 & Beta & Felici et al., 2014 \\
\hline \multicolumn{5}{|l|}{ Total procedure costs } \\
\hline SAN transfer (\$) & 8071.79 & 1614.36 & Gamma & MFFS payment values \\
\hline ICN transfer $(\$)$ & $10,918.02$ & 2183.60 & Gamma & MFFS payment values \\
\hline FFMT via SAN (\$) & $21,717.91$ & $21,718.11$ & Gamma & MFFS payment values \\
\hline
\end{tabular}

BLS = Bureau of Labor Statistics; MFFS = Medicare fee-for-service; $S D$ = standard deviation.

were QALYs associated with each MRC grade. This decision was made to prevent probabilistic scenarios in which patients with MRC Grade 0-1 had a higher QALY than patients with MRC Grade 4-5 simply because of the high variance among the reported values within the literature. ${ }^{1}$ Other than for QALYs, standard deviations for a given parameter were collected from the literature and modeled within the PSA, and when not available, a $20 \%$ standard deviation was used to model variation.

\section{Results}

\section{Base Case Analysis}

The base case model demonstrated a lifetime QALY of 22.45 in the SAN group, 22.0 in the ICN group, 22.3 in the FFMT group, and 21.3 in the no-treatment group. Given the improvements in QALY in the surgical groups compared with the no-treatment group, the base case model demonstrates that the 3 surgical interventions for PBPI were effective in improving QALYs across a lifetime. The lifetime costs of income lost through disability and interventional/ rehabilitation costs were $\$ 683,400$ in the SAN group, $\$ 727,400$ in the ICN group, $\$ 704,900$ in the FFMT group, and $\$ 783,700$ in the no-treatment group. These findings indicate that the 3 surgical strategies not only improved QALY after intervention, but over a lifetime each of these treatment strategies were cost saving, despite the costs of surgery, through the increased likelihood of returning to work and recovering income. This model demonstrated that the SAN transfer was the most cost-effective strategy dominating ICN transfer, FFMT, and no treatment (Fig. 2). ICN transfer, FFMT, and no treatment each yielded negative ICERs of \$255,113/QALY gained, \$105,214/QALY gained, and \$97,500/QALY gained, respectively. These negative ICERs indicate that these treatment strategies resulted in greater costs over a lifetime for less QALY and that the more cost-effective SAN dominated these strategies. Comparing the cost-effectiveness of the SAN transfer with the no-treatment arm directly, patients who chose no treatment incurred $\$ 102,700$ additional cost over a lifetime through lost income related to disability and an additional loss of 1.05 QALYs over a lifetime due to less quality of life with MRC Grade 0-1 elbow flexion.

\section{One-Way Sensitivity Analysis}

The cost-effectiveness model was sensitive to the rates of achieving good outcome of MRC Grade 4-5 after the 3 procedures. Using the variable success rates within the literature, a 1-way sensitivity analysis was performed to model the range of good outcome for SAN transfers, ICN transfers, and FFMT across the literature (Table 3). As 


\section{A. R. Wali et al.}

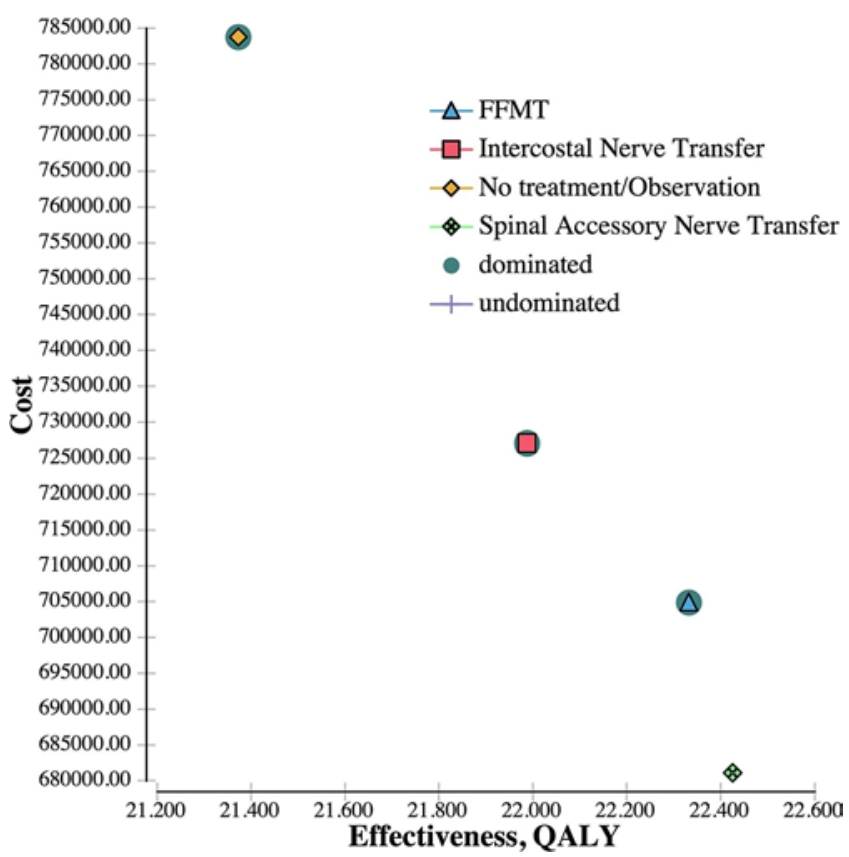

FIG. 2. This cost-effectiveness chart demonstrates overall costs and QALYs associated with the 4 management options. SAN transfer is the most cost-effective option, providing the greatest QALY over a lifetime while providing the lowest lifetime costs. No treatment (yellow and highlighted) is dominated by the 3 surgical interventions available to restore elbow flexion.

the probability of achieving MRC Grade 4-5 ranged from $29 \%{ }^{19}$ to $43 \%,{ }^{30}$ SAN nerve transfer dominated as the costeffective strategy for surgical success rates greater than $34 \%$. When modeling the range of surgical success rates of ICN to achieve MRC Grade $4-5$ from $20 \%{ }^{30}$ to $41 \%{ }^{19}$ FFMT and SAN transfer still remained more cost-effec- tive options. When modeling the range of good outcome for FFMT from $39 \%$ to $50 \%,{ }^{32}$ FFMT was the dominant strategy for success rates greater than $48 \%$. The notreatment strategy failed to become cost-effective even at the lowest rates of surgical success of the 3 interventions. One-way sensitivity analysis demonstrated that across a range of total costs for SAN transfer and FFMT, surgery remained more cost-effective than no treatment for total costs exceeding $\$ 100,000$. ICN transfer remained cost-effective relative to the no-treatment strategy until surgical costs exceeded $\$ 70,000$.

\section{Two-Way Sensitivity Analysis}

To directly compare changes in surgical success rates across two modalities at the same time, 2-way sensitivity analyses were constructed (Fig. 3). FFMT was more cost-effective than SAN transfer when SAN transfer success rates fell below 33\%, or if FFMT success rates exceeded $49 \%$. ICN transfer was more cost-effective than both FFMT and SAN transfer when ICN had success rates greater than $33 \%$, while SAN transfer had success rates less than $32 \%$.

\section{Probabilistic Sensitivity Analysis}

The Monte Carlo PSA, which used distributions of all input variables (such as cost of surgery, probability of surgical success, and returning to work after successful surgery) to account for parameter variability across 100,000 iterations, demonstrated that SAN transfer dominated as the most cost-effective strategy (Fig. 4). As willingness-topay continued to rise, FFMT had an increased likelihood of being a cost-effective strategy. At a willingness-to-pay cutoff of $\$ 50,000$, SAN transfer dominated in $88.5 \%$ of iterations, FFMT dominated in $7.5 \%$ of iterations, ICN dominated in $3.5 \%$ of iterations, and no treatment dominated in $0.5 \%$ of iterations.

TABLE 3. One-way sensitivity analysis demonstrating changes in ICER

\begin{tabular}{cccccc}
\hline \multirow{2}{*}{$\begin{array}{c}\text { Probability of MRC Grade } \\
\text { 4-5 for Elbow Flexion }\end{array}$} & $\begin{array}{c}\text { \% Used for Sensitivity } \\
\text { Analysis }\end{array}$ & \multicolumn{2}{c}{ ICER for Transfer (\$/QALY Gained) } & Cost-Effective \\
\cline { 3 - 4 } w/ SAN transfer & 29 & $-15,531$ & $-64,360$ & 0 & FFMT \\
& 32 & 0 & $-64,360$ & 23,900 & FFMT \\
& 35 & 0 & $-64,360$ & 129,411 & SAN \\
& 37 & 0 & $-64,360$ & $1,322,256$ & SAN \\
& 40 & 0 & $-107,700$ & $-445,012$ & SAN \\
w/ ICN transfer & 43 & 0 & $-105,300$ & $-255,113$ & SAN \\
& 20 & 0 & $-105,034$ & $-255,113$ & SAN \\
& 24 & 0 & $-105,673$ & $-255,113$ & SAN \\
& 28 & 0 & $-106,663$ & $-255,113$ & SAN \\
& 33 & 0 & $-108,400$ & $-255,113$ & SAN \\
w/ gracilis FFMT & 37 & 0 & $-112,248$ & $-255,113$ & SAN \\
& 41 & 0 & $-128,004$ & $-255,113$ & SAN \\
\hline & 39 & 0 & $-105,308$ & $-255,113$ & SAN \\
& 41 & 0 & $-105,208$ & $-365,565$ & SAN \\
& 43 & 0 & $-105,208$ & $-1,048,398$ & SAN \\
& 46 & 0 & $-73,935$ & 492,756 & SAN \\
& 48 & 0 & $-76,129$ & 124,250 & SAN \\
& 50 & 0 & $-78,006$ & $37,686.24$ & FFMT \\
\hline
\end{tabular}

Strategies are not considered cost-effective if they result in negative ICERs or a positive ICER $>\$ 50,000 / Q A L Y$ gained. 


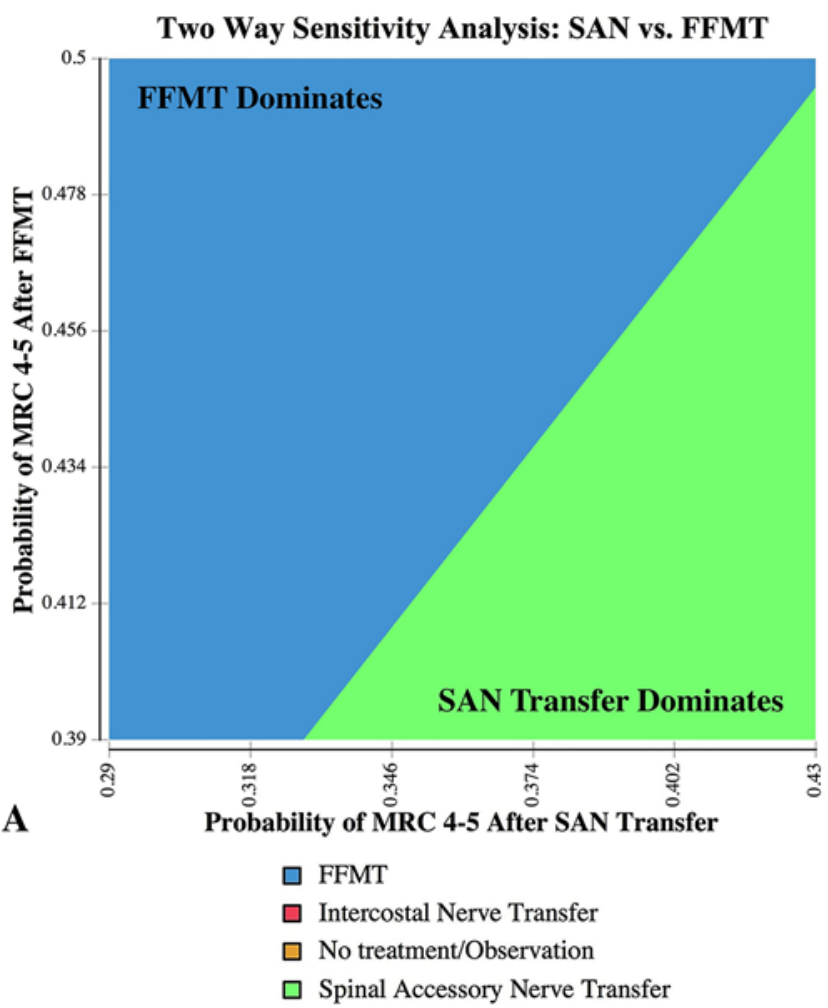

Two Way Sensitivity Analysis: SAN Transfer vs. ICN Transfer

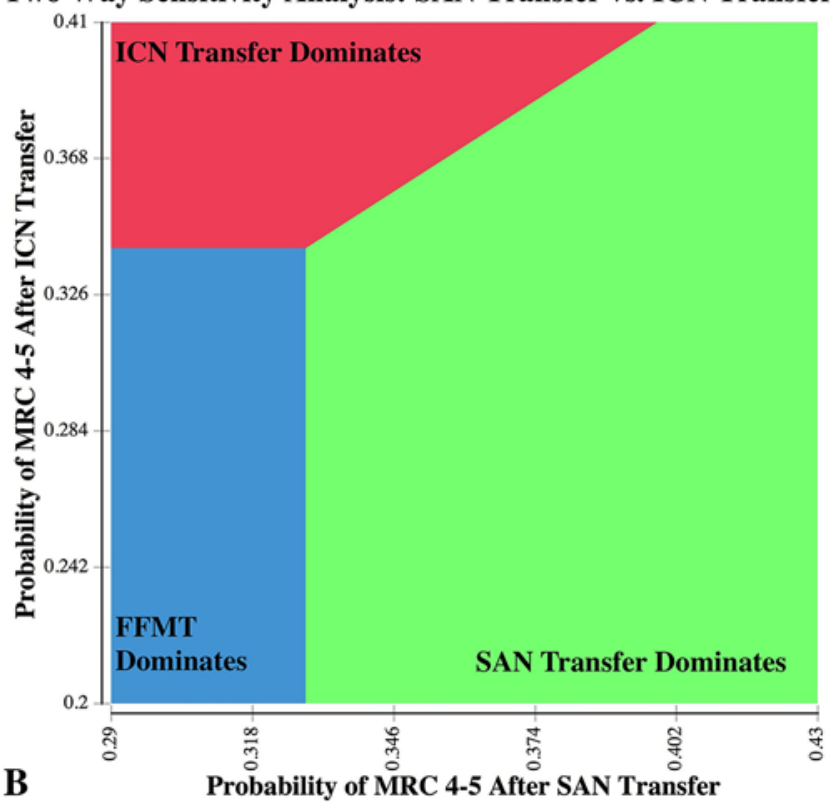

FIG. 3. Two-way sensitivity analyses were constructed to compare ranges of surgical success rates between 2 competing interventions. A: FFMT dominated SAN transfer as the cost-effective strategy as SAN transfer success rates fell below $33 \%$, or if FFMT success rates exceeded 49\%. B: ICN transfer dominated both FFMT and SAN transfer as the cost-effective strategy if ICN had success rates greater than $33 \%$ while SAN transfer had success rates less than $32 \%$.

\section{Discussion}

The role of reconstructive neurosurgery in restoring functionality and improving quality of life in patients who have sustained nerve injury not amenable to spontaneous

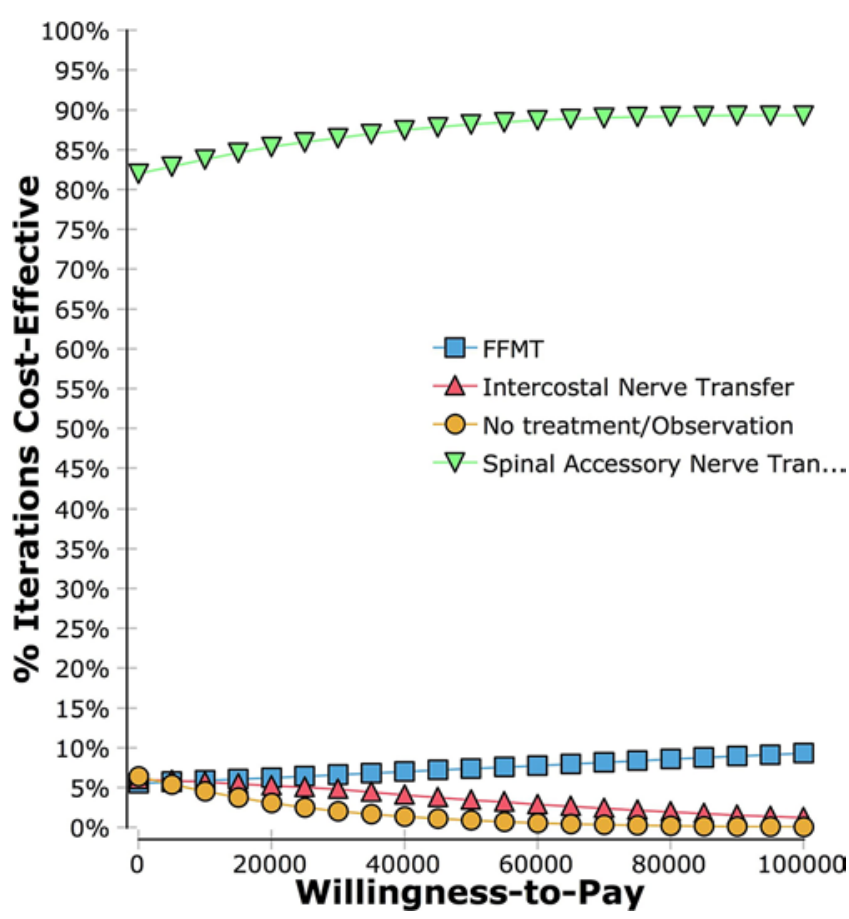

FIG. 4. This PSA demonstrates that across a broad range of willingnessto-pay, SAN transfer remained the dominant cost-effective strategy. At a willingness-to-pay cutoff of $\$ 50,000$, SAN transfer dominated in $88.5 \%$ of iterations, FFMT dominated in $7.5 \%$ of iterations, ICN transfer dominated in $3.5 \%$ of iterations, and no treatment dominated in $0.5 \%$ of iterations.

recovery continues to expand as new modalities, procedures, and techniques are available to patients. Numerous nerve transfers exist through manipulation of redundancies within the peripheral nervous system to restore functionality. Muscle transplants also provide options for patients who have chronic denervation of a muscle and offer treatment options for patients who did not undergo immediate nerve transfer surgery.

A prior cost-effectiveness study has demonstrated the efficacy of nerve transfer surgery to restore elbow flexion for patients with upper brachial plexus injury. ${ }^{31}$ This present work expands upon this literature and addresses the cost-effectiveness of the role of timing and treatment strategy to manage persistent PBPI with C5-T1 involvement. If patients are unable to undergo nerve transfer surgery within the critical window of approximately 6 months after injury, the likelihood of restoring functionality after a nerve transfer becomes increasingly small. For these patients, a muscle transplant is an excellent option to restore functionality. Moreover, this model demonstrates that patients who undergo early nerve transfer surgery either through SAN or ICN or undergo a muscle transplant will experience dramatic improvements in quality of life and have an increased likelihood of returning to work and recovering income. For this reason, although there are clear costs with intervention, these treatment strategies can be considered cost saving across a lifetime.

While the base case model demonstrated that SAN transfer was the most cost-effective strategy, the 1- and 2 -way sensitivity analysis demonstrated that variations in 
the success rate for a given surgery offer variability among the surgical strategies. This model demonstrates that as SAN transfer success rates for MRC Grade 4-5 exceeded $34 \%$, this modality was more cost-effective than ICN transfer or FFMT. As success rates for FFMT exceeded $48 \%$ or as SAN success rates dropped below $33 \%$, FFMT could dominate SAN transfer. However, even at the lowest success rates of any of the surgical interventions, the notreatment strategy failed to become a cost-effective strategy. These findings were further substantiated by the PSA that demonstrated that SAN transfer dominated $88.5 \%$ of iterations, ICN transfer dominated in $3.5 \%$ of iterations, and FFMT dominated in $7.5 \%$ of iterations, and no treatment dominated in $0.5 \%$. These findings illustrate that surgical intervention, either through nerve transfer surgery or through muscle transplantation, is effective in improving quality of life. Importantly, the no-treatment arm was a cost-effective strategy in less than $1 \%$ of iterations, indicating the urgent and valuable role of reconstructive neurosurgery for patients with PBPI. Even if patients have missed the optimal treatment window to have nerve transfer surgery, this model provides clear evidence that muscle transplantation can excellently improve functionality and quality of life.

This model has limitations. This model focuses on the restoration of elbow flexion as a means of improving quality of life and improving the rates with which patients are able to return to the workforce and recover income. This model does not address the role of nerve transfer surgery to restore shoulder abduction or the role of the double FFMT to restore elbow flexion and hand flexion simultaneously. ${ }^{11,15}$ The therapeutic options available to patients to restore additional functionality within the hands or to restore sensation vary broadly and are beyond the scope of this analysis. While this model focuses on FFMT as a delayed therapeutic option, some centers perform FFMT within the first year of injury. ${ }^{2}$ This model would suggest that those interventions may also be cost-effective and clinically appropriate. Many of the surgical success rates used within this model are collected from single-center published data. To address this limitation, 1- and 2-way sensitivity analyses were conducted to assess ranges in surgical success rate to capture variation in technical performance among the treatment modalities. The costs incorporated within this model were derived from Medicare data and may not represent the costs within all health care systems, all types of costs associated with diverse insurance coverage options, or costs associated with additional physical therapy and subsequent rehabilitation. To address this limitation, 1-way sensitivity analysis was performed to establish that SAN transfer and FFMT were still more cost-effective than no treatment for total costs exceeding $\$ 100,000$ and that ICN transfer was cost-effective compared with no treatment until total costs exceeded $\$ 70,000$. With regard to rehabilitation time, the reported literature describes that rehabilitation time for FFMT is typically less than 1 year ${ }^{4}$ and rehabilitation time for nerve transfer surgery can range from less than 1 year to 2 years. ${ }^{30}$ Our model makes the assumption that rehabilitation time is the same between both of these procedures and captures indirect costs associated with rehabilitation and functional status through assessing the proportion of patients who are able to return to the workforce after surgery.

Despite these limitations, this model is the first costeffectiveness analysis to address the management of PBPI by analyzing the competing management strategies of SAN transfer, ICN transfer, delayed gracilis FFMT, and no treatment. These findings should be considered in determining the ideal management options for patients who have sustained PBPI to optimize quality of life, restore functionality, minimize surgical costs, and increase the likelihood of helping patients achieve functional independence.

\section{Conclusions}

Nerve transfer surgery and muscle transplantation remain critical tools available to surgeons to restore elbow flexion, improve quality of life, and increase the likelihood of patients returning to work after sustaining PBPI. This cost-effectiveness model assesses the competing treatment strategies available to patients and demonstrates that patients should undergo treatment with nerve transfer surgery or FFMT to restore functionality. For patients within the 6-month window after injury, this model suggests that SAN transfer may be the more cost-effective strategy. For patients outside of the 6-month window, FFMT remains a cost-effective and important intervention to restore elbow flexion functionality to patients. This analysis should inform medical decision makers and patients alike to use these restorative neurosurgical interventions for this patient population.

\section{Acknowledgments}

Mr. Wali is funded through an NIH TL1 predoctoral grant (no. 1TL1TR001443).

\section{References}

1. Ali ZS, Bakar D, Li YR, Judd A, Patel H, Zager EL, et al: Utility of delayed surgical repair of neonatal brachial plexus palsy. J Neurosurg Pediatr 13:462-470, 2014

2. Barrie KA, Steinmann SP, Shin AY, Spinner RJ, Bishop AT: Gracilis free muscle transfer for restoration of function after complete brachial plexus avulsion. Neurosurg Focus 16(5):E8, 2004

3. Belzberg AJ, Dorsi MJ, Storm PB, Moriarity JL: Surgical repair of brachial plexus injury: a multinational survey of experienced peripheral nerve surgeons. J Neurosurg 101:365-376, 2004

4. Bishop AT: Functioning free-muscle transfer for brachial plexus injury. Hand Clin 21:91-102, 2005

5. Braithwaite RS, Meltzer DO, King JT Jr, Leslie D, Roberts MS: What does the value of modern medicine say about the $\$ 50,000$ per quality-adjusted life-year decision rule? Med Care 46:349-356, 2008

6. Cho AB, Paulos RG, de Resende MR, Kiyohara LY, Sorrenti L, Wei TH, et al: Median nerve fascicle transfer versus ulnar nerve fascicle transfer to the biceps motor branch in C5-C6 and C5-C7 brachial plexus injuries: nonrandomized prospective study of 23 consecutive patients. Microsurgery 34:511-515, 2014

7. Compston A: Aids to the investigation of peripheral nerve injuries. Medical Research Council: Nerve Injuries Research Committee. His Majesty's Stationery Office: 1942; pp. 48 (iii) and 74 figures and 7 diagrams; with aids to the examination 
of the peripheral nervous system. By Michael O'Brien for the Guarantors of Brain. Saunders Elsevier: 2010; pp. [8] 64 and 94 Figures. Brain 133:2838-2844, 2010

8. Concato J, Feinstein AR: Monte Carlo methods in clinical research: applications in multivariable analysis. J Investig Med 45:394-400, 1997

9. Dasta JF, McLaughlin TP, Mody SH, Piech CT: Daily cost of an intensive care unit day: the contribution of mechanical ventilation. Crit Care Med 33:1266-1271, 2005

10. DiTaranto P, Campagna L, Price AE, Grossman JA: Outcome following nonoperative treatment of brachial plexus birth injuries. J Child Neurol 19:87-90, 2004

11. Doi K, Muramatsu K, Hattori Y, Otsuka K, Tan SH, Nanda V, et al: Restoration of prehension with the double free muscle technique following complete avulsion of the brachial plexus. Indications and long-term results. J Bone Joint Surg Am 82:652-666, 2000

12. Felici N, Zaami S, Ciancolini G, Marinelli E, Tagliente D, Cannatà C: Cost analysis of brachial plexus injuries: variability of compensation by insurance companies before and after surgery. Handchir Mikrochir Plast Chir 46:85-89, 2014

13. Franzblau LE, Maynard M, Chung KC, Yang LJS: Medical treatment decision making after total avulsion brachial plexus injury: a qualitative study. J Neurosurg 122:1413-1420, 2015

14. Giuffre JL, Kakar S, Bishop AT, Spinner RJ, Shin AY: Current concepts of the treatment of adult brachial plexus injuries. J Hand Surg Am 35:678-688, 2010 (Erratum in J Hand Surg Am 35:1226, 2010)

15. Hattori Y, Doi K, Ikeda K, Pagsaligan JM, Watanabe M: Restoration of prehension using double free muscle technique after complete avulsion of brachial plexus in children: a report of three cases. J Hand Surg Am 30:812-819, 2005

16. Hunink MG: In search of tools to aid logical thinking and communicating about medical decision making. Med Decis Making 21:267-277, 2001

17. Kim DH, Cho YJ, Tiel RL, Kline DG: Outcomes of surgery in 1019 brachial plexus lesions treated at Louisiana State University Health Sciences Center. J Neurosurg 98:1005-1016, 2003

18. Maldonado AA, Kircher MF, Spinner RJ, Bishop AT, Shin AY: Free functioning gracilis muscle transfer versus intercostal nerve transfer to musculocutaneous nerve for restoration of elbow flexion after traumatic adult brachial pan-plexus injury. Plast Reconstr Surg 138:483e-488e, 2016

19. Merrell GA, Barrie KA, Katz DL, Wolfe SW: Results of nerve transfer techniques for restoration of shoulder and elbow function in the context of a meta-analysis of the English literature. J Hand Surg Am 26:303-314, 2001

20. Midha R: Epidemiology of brachial plexus injuries in a multitrauma population. Neurosurgery 40:1182-1189, 1997

21. Moiyadi AV, Devi BI, Nair KPS: Brachial plexus injuries: outcome following neurotization with intercostal nerve. J Neurosurg 107:308-313, 2007

22. Nagano A: Treatment of brachial plexus injury. J Orthop Sci 3:71-80, 1998
23. Ray WZ, Mackinnon SE: Clinical outcomes following median to radial nerve transfers. J Hand Surg Am 36:201-208, 2011

24. Sakellariou VI, Badilas NK, Stavropoulos NA, Mazis G, Kotoulas HK, Kyriakopoulos S, et al: Treatment options for brachial plexus injuries. ISRN Orthoped 2014:314137, 2014

25. Samardzic M, Grujicic D, Antunovic V: Nerve transfer in brachial plexus traction injuries. J Neurosurg 76:191-197, 1992

26. Samii A, Carvalho GA, Samii M: Brachial plexus injury: factors affecting functional outcome in spinal accessory nerve transfer for the restoration of elbow flexion. J Neurosurg 98:307-312, 2003

27. Silva GB, Alves JA, Pisani MJ, da Costa RI, Rezende MR, Cho AB: Which nerve to use? Comparison between donor nerves to motorize the free functional gracilis muscle transfer for elbow flexion in traumatic brachial plexus injuries. J Hand Surg Am 41 (9 Suppl):S15-S16, 2016 (Abstract)

28. Torrance GW, Feeny D: Utilities and quality-adjusted life years. Int J Technol Assess Health Care 5:559-575, 1989

29. van der Ploeg RJ, Oosterhuis HJ, Reuvekamp J: Measuring muscle strength. J Neurol 231:200-203, 1984

30. Waikakul S, Wongtragul S, Vanadurongwan V: Restoration of elbow flexion in brachial plexus avulsion injury: comparing spinal accessory nerve transfer with intercostal nerve transfer. J Hand Surg Am 24:571-577, 1999

31. Wali AR, Park CC, Brown JM, Mandeville R: Analyzing cost-effectiveness of ulnar and median nerve transfers to regain forearm flexion. Neurosurg Focus 42(3):E11, 2017

32. Yang Y, Yang JT, Fu G, Li XM, Qin BG, Hou Y, et al: Functioning free gracilis transfer to reconstruct elbow flexion and quality of life in global brachial plexus injured patients. Scientif Report 6:22479, 2016

\section{Disclosures}

The authors report no conflict of interest concerning the materials or methods used in this study or the findings specified in this paper.

\section{Author Contributions}

Conception and design: Mandeville, Wali, Brown. Acquisition of data: Mandeville, Wali, Brown. Analysis and interpretation of data: all authors. Drafting the article: all authors. Critically revising the article: all authors. Reviewed submitted version of manuscript: all authors. Approved the final version of the manuscript on behalf of all authors: Mandeville. Statistical analysis: all authors. Administrative/technical/material support: all authors. Study supervision: all authors.

\section{Correspondence}

Ross Mandeville, Department of Neurological Surgery, University of California, San Diego, 200 West Arbor Dr., MC 8893, San Diego, CA 92103.email: rmandeville@ucsd.edu. 